\title{
Paved with Bad Intentions: QAnon's Save the Children Campaign
}

\author{
Cody Buntain, Mila Johns, Monique Deal Barlow, and Mia \\ Bloom
}

\begin{abstract}
In the lead-up to the 2020 US presidential election, adherents to QAnon conspiracy theories parlayed the "Save the Children" slogan from a well-known charity into a vector for exposure and political mobilization. This paper introduces three factors that potentially contributed space for appropriating the slogan into the QAnon-backed \#SaveTheChildren campaign and, in this context, examines images of children shown alongside QAnon \#SaveTheChildren messages. For these factors, we examine the use of race, age, gender, and graphic depictions in this exploitative imagery. Results show QAnon-related images massively overrepresent preteen, white children compared to child-trafficking statistics and include a substantial proportion of graphic imagery. Building on studies of child-advocacy campaigns, we contend that QAnon imagery uses the familiar motif of distressed children while presenting a narrative distinct from both these child-advocacy groups and the realities of child trafficking. Embedded in broader contexts of race, gender, and emotional manipulation in online spaces, we discuss how these images are particularly impactful for conservative, white, and especially female audiences in the US and how other movements might be at similar risk of appropriation. The paper then closes by describing possible interventions to protect the safety of online audiences.
\end{abstract}

\section{Introduction}

In the summer of 2020, QAnon conspiracy theorists hijacked the \#SaveTheChildren hashtag campaign, parlaying the name of a well-known charity into a springboard for exposing new audiences to QAnon messaging (Roose 2020a). Beyond potentially revitalizing the QAnon movement (Roose 2020a), QAnon's appropriation of this campaign has created significant problems for legitimate anti-trafficking organizations, strained law enforcement with false "tips" about trafficked children, and undermined the fundraising efforts of legitimate charities dedicated to child protection (Seitz 2020). Worse still, QAnon conspiracy theorists have suggested these charitable organizations are actively involved in the trafficking and exploitation of children. Save the Children, the actual charity, has consequently used resources needed elsewhere to clarify that it is unaffiliated 
with these campaigns (Save the Children 2020). The Polaris Project advocacy group has even stated that the effort spent responding to such false reports in 2020 "could have been used to respond to an additional 42 trafficking cases" (Rajan et al. 2021). These effects are not limited to negative impacts on charities, as surveys of QAnon adherents on Telegram show a desire to "save the children" as the most common attraction to the movement (Garry et al. 2021), suggesting this appropriated campaign has been a successful recruiting tool for the QAnon movement. It is in this context that this paper examines how the QAnon movement has appropriated this hashtag campaign, where we focus specifically on QAnon-related depictions of children in visual media.

We develop this characterization by examining the age, gender, ethnicity, and wellbeing of children in visual media posted alongside QAnon's \#SaveTheChildren campaign. Connecting these depictions to research on social media mobilization, gender politics, and early narratives used by child-advocacy organizations, we posit that the success of this QAnon campaign stems from a confluence of factors: First is the appropriation of the children-in-need motif, which has been used by charity and religious organizations for decades. Second is the mobilizing effects of fear- and anger-evoking images, which graphic depictions of children in distress can activate. And third is this campaign's use of these visuals to exploit white fear and gender politics in US audiences. We use these factors to develop several hypotheses about QAnon imagery, which we evaluate through qualitative and comparative assessment.

Our results show that, while QAnon imagery has a modicum of grounding in the truth of child trafficking, these images present a largely warped reality of this issue, especially around race and age. We see that QAnon's \#SaveTheChildren imagery reflects the US-national and global statistics of female-presenting children being victims of child trafficking but departs from these statistics in their depictions of younger children. That is, QAnon media predominantly depicts preteen children despite actual trafficking statistics being more equally split between preteen and teenage children. We also find that racial depictions in QAnon imagery depart significantly from the reality of child trafficking in the US and abroad: Specifically, depictions of white children are massively overrepresented in QAnon imagery compared to actual incidences of child trafficking, and these white children are more often depicted in graphic situations.

Through these analyses, this work contributes a better understanding of QAnon's coopted \#SaveTheChildren hashtag campaign and its use of the visual medium for recruitment. As the QAnon movement has already been linked to online polarization and offline violence (Garry et al. 2021), these characterizations have implications for both online and real-world safety. We close this work with a discussion of these implications, including potential counter-messaging strategies and how these depictions of children may be particularly activating among conservative, female audiences-as supported by accounts of gender in the larger QAnon movement (Argentino and Crawford 2021; Bracewell 2021) and QAnon's relationship with mothers (Kelly 2020).

\section{Background and Related Work}

This article's central question asks why QAnon has been so successful in appropriating the \#SaveTheChildren hashtag and slogan to reach and mobilize new followers. We posit that this success stems in part from a confluence of three factors: 1) QAnon's campaign leverages a familiar visual motif often used by child-advocacy groups (though the particular images used by QAnon might be quite different from advocacy groups' images), 2) fear- and anger-evoking images have known mobilizing qualities on social 
media, and 3) QAnon imagery exploits white audiences' racialized fears of attacks on white families.

In the sections below, we first outline the history and evidence of this co-opted campaign and then connect prior research on these three factors. We convert these connections into a set of hypotheses about the depictions of children we expect to find within QAnon imagery and how they relate to actual incidence of child trafficking.

\subsection{QAnon: A Primer and the Appropriation of \#SaveTheChildren}

QAnon is a baseless conspiracy theory predicated on the belief that a secret cabal of child-abusing pedophiles runs the world (Forrest 2021; Hannah 2021). This cabal is generally depicted as a group of "nefarious elites," dominated by celebrities such as Tom Hanks, Oprah Winfrey, Chrissy Teigen, and the Catholic Church's Pope Francis (Heffernan 2020). QAnon adherents believe that this cabal's members are blood-drinking pedophiles, and the images QAnon followers use often portray children abducted, beaten, and exploited for their blood and sexual abuse. The movement has its origins in the anonymous online messaging board 4chan, where an individual going by the pseudonym "Q" shared messages about a secret government program to identify and prosecute members of this global cabal. These messages would often include predictions about a "Gathering Storm" or "Great Awakening," wherein this global cabal would finally be exposed for its crimes. Despite virtually all of Q's predictions about this storm failing to materialize (Zuckerman 2019; Roose 2021), the conspiracy theory and movement around it continued to grow, becoming a central topic in US and international politics (Hoseini et al. 2021).

A particularly concerning aspect of this growth is the movement's ability to subsume many other conspiracies into its narrative, thereby contributing to its international growth and virality. In form, the QAnon conspiracy theory parallels moral panic and many Satanic conspiracy theories and calls upon its adherents to fight against the forces of evil (Vrzal 2020). As the QAnon conspiracy is particularly appealing to Republicans (Civiqs 2020), their "ideology" has been used to mobilize conspiracy theorists around political figures such as Donald Trump and General Michael Flynn. For a more in-depth discussion of QAnon's tenets and adherents, see Bloom and Moskalenko 2021 and Moskalenko and McCauley 2021.

Concerns around violence and radicalization linked to QAnon content have prompted several online social media platforms and technology companies to ban or suppress QAnon-related content. For example, Facebook's policies about how it deals with violent movements and organizations features references to QAnon, and the company has taken steps to suppress QAnon content shared on its platforms that violate its terms of service (Facebook 2021). Likewise, Reddit banned QAnon-related communities in September 2018 (Papasavva et al. 2021), and 8chan-a 4chan clone popular among QAnon adherents-was taken offline by CloudFlare in 2019 (Roose 2019). Twitter has taken similar steps in July 2020 (Conger 2020) and again in October 2020 and January 2021 (Conger 2021).

A Co-opted Charity Campaign In the wake of increasingly punitive measures by social media platforms against QAnon content sharing in the summer of 2020, the QAnon movement needed new avenues to expand its audience. Given the movement's foundation of anti-elite and anti-pedophilia messaging and its history of exploiting fears and societal ills, the 2020 political climate was rife with opportunities for new messaging. Conspiracies around sex trafficking were prominent, especially around Wayfair, a global furniture company falsely accused of trafficking children through its website (McNeal 
2020), and continued theorizing around Jeffrey Epstein's death-an American financier who died in 2019 in his jail cell after being arrested on federal charges of sex trafficking of minors-and his connections to political elites.

At the same time, the prominent international charity and child-advocacy organization "Save The Children" has used online hashtag campaigns to raise awareness and solicit donations, often using a hashtag of the group's name, \#SaveTheChildren, to amplify their messaging. A search for tweets from the organization that include the \#SaveTheChildren hashtag produces 16 tweets between February 2011 and January 2019, where the most engaging message received 221 retweets and 240 "like" reactions; on average, each of these tweets received 37 retweets and 34 "like" reactions. Similarly, the United Kingdom branch of the Save The Children charity has used this hashtag 199 times since June 2010, also averaging about 3 retweets per tweet and 5 "like" reactions per tweet.

As a leading charity with a 100-year track record of helping children, this organization has well-established visibility in the child-advocacy space and would be familiar to many Internet users. Leading up to the summer of 2020 , the charity had successfully used hashtag campaigns to raise funds. For example, in 2018, the \#foreverychild hashtag campaign amplified the success stories of educating girls and preventing early marriage, \#peaceday emphasized their post-conflict reconstruction work, and \#protecteducation brought attention to and tried to prevent schools from being used by militants. In fact, social media has proven a valuable channel for organizations like Save the Children to engage with donors (Guo and Saxton 2018; Lovejoy and Saxton 2012).

In the summer of 2020, however, the \#SaveTheChildren hashtag campaign experienced a surprising swell of popularity. In early August, Facebook posts including this hashtag saw a sixtyfold increase in average weekly engagement compared to weekly engagement over the preceding year (Rogers 2020). Troubling QAnon-style talking points began to appear alongside messages with the \#SaveTheChildren and related hashtags, twisting the message about child trafficking to include references to celebrities and political elites (Roose 2020b; Petrosky 2020; Rogers 2020). QAnon supporters began to flood Facebook and Instagram with posts about human trafficking and child rape, spreading into parenting groups, and by the end of the summer, researchers had identified over 100 Facebook groups that were pushing a combination of anti-trafficking messages related to \#SaveTheChildren and QAnon content (ibid). These groups also saw a thirtyfold increase in membership (Roose 2020a). This \#SaveTheChildren campaign was not limited to Facebook, with the hashtag seeing traction across other social media platforms, especially Twitter (Roose 2020b) and Instagram (Bracewell 2021). Researchers have since credited QAnon's appropriation of the \#SaveTheChildren campaign with mobilizing followers, especially women (Bracewell 2021; Kelly 2020; Dickinson 2020; Roose 2020b), and revitalizing the movement (Roose 2020a). This success was also particularly evident in Garry et al. 2021, where "saving the children" was the most commonly cited attraction in surveys of QAnon adherents on Telegram.

When the QAnon campaign used the charity's name, it created significant problems for legitimate anti-trafficking organizations. On the one hand, several child-advocacy organizations saw a significant increase in donations following the popularity of the \#SaveTheChildren campaign (Rogers 2020). On the other hand, the increase in paranoia around child trafficking strained law enforcement with false "tips" about trafficked children and undermined the fundraising efforts of legitimate charities dedicated to child protection (Seitz 2020). Worse still, QAnon conspiracy theorists have suggested that charitable organizations (like Save the Children and UNICEF) were actively involved in the trafficking and exploitation of children. As a result of the QAnon campaign, the charity Save the Children subsequently received hundreds of threatening phone calls and voice mails. The Polaris Project advocacy group has even stated that the effort 
spent responding to such false reports in 2020 "could have been used to respond to an additional 42 trafficking cases" (Rajan et al. 2021).

\subsection{Appropriation and a Confluence of Three Factors}

While it is likely incorrect to ascribe a single overarching and coherent strategy to the QAnon \#SaveTheChildren campaign, its pseudo-organic popularity, appropriation of well-intentioned online campaigns, and the resulting exposure of many otherwise naive individuals to QAnon tenets represent a concern for online safety. To prevent or mitigate future instances of such appropriation, one must first understand the factors that provided space for this takeover to occur. Below, we outline three potential driving factors that contributed the space necessary for the QAnon movement to co-opt the \#SaveTheChildren campaign so successfully.

Factor 1: Foundations and Visual Motifs Around Child Trafficking A common tactic for social media marketing campaigns is to integrate current events and engage with existing societal topics (Borah et al. 2020)-e.g., Bonaparte 2020 discusses corporate marketing strategies and rebranding efforts in support of African American communities following the murder of George Floyd. Malevolent influence campaigns have adopted similar strategies, as evidenced by studies of Russian disinformation, where campaigns proved particularly adept at leveraging the "Black Lives Matter" movement and issues of racial justice in the US (Arif, Stewart, and Starbird 2018). Similarly, as shown in Freelon et al. 2020, inauthentic "troll" accounts were more effective at garnering engagement when they presented themselves as "Black activists," illustrating both a key racial component in online influence and a willingness to exploit racial divides in the US for political gain. In this context, child trafficking and sexual abuse yield exploitable social ills.

Because trafficking is an ongoing societal problem (Bigio and Ortiz 2021), a movement like QAnon-already adjacent to child-trafficking issues given its foundation in conspiracies of global networks of pedophilia-can engage with existing conversations and audiences already interested in this topic. By associating its messaging with an extant organization like Save the Children and its campaigns, QAnon messaging can imbue itself with legitimacy, as the problem of child trafficking is in fact real and urgent. As one in four victims of modern slavery are children, meaning that some 10 million children are enslaved (UNODC 2020), this grain of truth provides a foundation for QAnon messaging, as "Everyone agrees that child trafficking is very bad, and the argument QAnon makes is, 'If you're against us talking about this, you're in favor of child trafficking'" (Roose 2020a).

Child trafficking is more than just a societal ill, however, as concerns around children in need are longtime societal issues. The "Save The Children" charity is a prime example of this issue's longevity, as it was founded in May 1919 to alleviate famine in AustriaHungary and Germany in the aftermath of World War I. Beyond the topic's age, though, is the medium through which issues of children's suffering have often been shared. Specifically, issues of child exploitation, starvation, and suffering have long been communicated via photographic media-e.g., a photo of Alan Kurdi, a drowned three-year-old Syrian boy during the 2015 refugee crisis (Dahmen, Mielczarek, and Morrison 2019), or Omran Daqneesh, a five-year-old Syrian boy who garnered international attention after being photographed in an ambulance following a Syrian-government airstrike (Wilson, Zhou, and Starbird 2018) - with such photos often rising to the level of "iconic imagery" (Dahmen, Mielczarek, and Morrison 2019). The commonality and impact of these photos of children suffering, we contend, establishes a visual motif that can be further exploited by malevolent groups, as audiences are already familiar with these graphical representations. Consequently, movements like QAnon can not only exploit the textual discussion 
around child trafficking but also further increase its impact by co-opting similar-and therefore familiar-visuals.

\section{Depictions of Children in Charitable Campaigns.}

QAnon imagery is not the first campaign to use depictions of children in distress. Charitable organizations have long used such imagery, often referred to as "poverty porn," to stimulate donations-"exploiting the poor in charity ads to generate sympathy to increase donations" (Wymer and Gross 2021). These depictions can stimulate a guilt response in the audience, thereby increasing donations, especially among female audiences (Rijn, Barham, and Sundaram-Stukel 2017). For example, foreign aid commercials in the past have featured celebrities like Sally Struthers advancing this message; in one advertisement, Struthers said, "Welcome to hell, hell on earth" as she stumbled through a Latin American slum. Despite the use of disturbing imagery, the former director of Christian Children's Fund (CCF) credited Struthers as the primary reason the charity's annual income soared from $\$ 29$ million in 1976 to $\$ 103$ million by 1991 (Zielinski and Jackson 1998). This poverty-centric approach to charitable giving was common in the past. Beginning in 1976, late-night commercials and advertising campaigns targeted viewers with graphic images as they entreated viewers to loosen the purse strings and sponsor a child for "the daily cost of a cup of coffee."

Despite this potentially positive impact of graphic material, the problematic nature of such depictions has moved aid organizations, as articulated in Lentfer 2018:

"Non-profit organisations have faced scrutiny about approaches that raise awareness and money, but do not invite the public to question why poverty exists in the first place...finding a balance between telling compelling stories, without trivialising people's lives, or the long-term prospects for social change."

Recently, however, charitable organizations like UNICEF, Save the Children, the Children's Defense Fund, and Children International are moving away from such depictions, instead featuring uplifting messages of empowerment and success. While charities might occasionally leverage pity, shame, or guilt, these evocations are no longer the principal approaches. Much of the messaging centers around making the donors feel good that their money is being put to good use and that their donations have helped achieve some positive goal. Studies have shown that guilt-inducing images are a poor long-term strategy even if they are successful in the short term, as potential donors become desensitized to guilt-based depictions (Rijn, Barham, and Sundaram-Stukel 2017).

In this context, the QAnon appropriation of \#SaveTheChildren can be viewed as paralleling historical campaigns. Despite this shift away from poverty porn, visuals of children in need persist, leaving an opportunity for QAnon to repurpose a visual that is sufficiently ingrained in popular consciousness. These common depictions of children in need can be described as recurrences of a visual motif (Yonetani, Kitani, and Sato 2016), or a distinctive recurring element that provides a "compact source of cultural information" (Yarlott and Finlayson 2016). The power of this motif is that, when an individual on social media is exposed to an image of a child in need accompanied with some call to action, the individual can quickly glean the meaning of such an image. We posit that, by exploiting this commonly occurring motif, the QAnon campaign can anchor itself on an existing, common cultural understanding and more easily access a receptive audience. 


\subsection{Factor 2: Mobilizing Effects of Negativity and Evocative Images on Social Media}

Early research into social media portrayed the platforms as mobilizing forces for democratization and social good, as argued during the Arab Spring and wave of public demonstrations that used social media to push for political reforms (Howard et al. 2011). While popular sentiment toward social media platforms has cooled substantially following concerns of online manipulation during the 2016 US presidential election (Auxier 2020) and revelations about private deals between Facebook and Jared Kushner (Chafkin 2021), studies continue to show social media has a powerful mobilizing effect (Brantly 2019; Enjolras, Steen-Johnsen, and Wollebæk 2013; Yamamoto, Kushin, and Dalisay 2015). Such mobilization is not specific to social media, though. As we discuss above, iconic imagery has power: Kurdi's death and its photographic depiction are thought to have had significant political impact in Europe, with popular sentiment pushing governments to increase assistance to migrants (Dahmen, Mielczarek, and Morrison 2019).

Specific to social media, however, studies have repeatedly shown that negative content consistently garners more online attention (Berger and Milkman 2012; Fan et al. 2014; Shaer 2014), and untruths and rumors tend to outpace facts (Vosoughi, Roy, and Aral 2018) and corrections (Arif et al. 2017). When that content is photographic in nature, recent studies show such imagery also has significant mobilizing power for public demonstration (Casas and Williams 2019). This appropriation and use of visual media is also of particular concern, as prior studies show that exposure to such imagery can serve to mobilize individuals to participate in physical demonstrations or violence (Casas and Williams 2019; Sánchez-Cuenca and Aguilar 2009). Such anger-inducing media may motivate online sharing more than other emotions, since "anger is a high-arousal emotion, which drives people to take action that makes you feel fired up, which makes you more likely to pass things on" (Shaer 2014). This robust finding holds across a variety of platforms (Berger and Milkman 2012; Fan et al. 2014).

As with depictions of children in need, similar exploitation of images in social media is not a new phenomenon. Lilleker, Veneti, and Jackson 2019 provide a compendium of research into visual communication, clarifying the case that the visual is an equally impactful form of political communication. In this vein, clear evidence exists for images on social media as a form of political manipulation: As with societal divides, Russian influence efforts in social media exploit images of real-world events and political demonstrations and are especially effective at disseminating political imagery (Zannettou et al. 2020). Likewise, Seo 2014 outlines the conflict between Israel and Hamas, with both sides leveraging different types of visuals as propaganda, and Hamas in particular leveraging emotional frames in its imagery. This body of work demonstrates that exploitation of visual media has a long history, and a recent history especially in online spaces, establishing a clear space for the QAnon movement to appropriate and manipulate such visuals.

This context suggests the QAnon \#SaveTheChildren campaign exists in a broader space of visual manipulation. Taken with the motif description above, these factors provide a cultural anchor from which the QAnon campaign can build, and by twisting these familiar depictions of suffering children into imagery that evokes intensely negative emotions, the campaign can access the mobilizing aspects of social media to entice individuals to share and amplify its messages. We posit that this goal of mobilization, however, is fundamentally different from the goals of imagery used by charitable organizations. While a charitable organization may use depictions of children in need, these organizations desire sustained mobilization and donation from their donors, as the move away from poverty porn-style depictions suggests, since its use hinders long-term action (Rijn, Barham, and Sundaram-Stukel 2017). In contrast, QAnon-related campaigns do not need 
this long-term action to spread their message; rather, if QAnon adherents can mobilize audiences to join their groups in the short term by relying on guilt- and anger-inducing imagery, the radicalization process can take over separately from these poverty-porn images.

\subsection{Factor 3: Exploiting Fears in White, Conservative Audiences}

The above two factors establish the message's form (i.e., familiar imagery of children in need) and its mechanism of mobilization (i.e., fear- and anger-inducing visuals), but an open question remains regarding the audiences targeted by or vulnerable to the QAnon \#SaveTheChildren campaign. Here, we posit that one of the main factors of this campaign is race and the exploitation of anxieties among white, conservative audiences. This racial component is based on prior work into online manipulation that shows race plays a distinct role (Freelon et al. 2020) and on studies of QAnon that reveal a consistent theme around racism (Papasavva et al. 2021). At the intersection of this racism, QAnon's conservative foundation, and anxieties about children's safety embodied in the \#SaveTheChildren campaign lies "white sexual politics." Bjork-James 2020 defines this term as a suite of concerns-especially salient among the religious right and white supremacists-in "defense of white racial privilege" and reinforcing heterosexual and patriarchal norms. This lens of white sexual politics suggests a potential identity for perpetrators depicted in the \#SaveTheChildren imagery: an "other," where this other may be foreign, non-white, non-heterosexual, or some other non-conforming identity. QAnon's central theme of a cabal of liberal elites that prey on children, reinforced by the \#SaveTheChildren imagery of children in distress, plays into these concerns. Prior work has shown that content that explicitly directs animosity toward these "others" or a similar out-group receives significantly more engagement in online spaces than other content (Rathje, Van Bavel, and Linden 2021). Consequently, if the QAnon campaign is meant to mobilize audiences, depictions that elicit fear of racialized "other" child abduction would likewise be a particularly strong activator among the religious right and white supremacists.

QAnon's appeal is not limited to these audiences (Zuckerman 2019; Petrosky 2020; Moskalenko and McCauley 2021; Bracewell 2021; Argentino and Crawford 2021), however; rather, it appears that QAnon's appropriation of the \#SaveTheChildren campaign has a significant draw among a less extreme, conservative audience as well (Roose 2020a; Garry et al. 2021).

We instead suggest the racial anxieties we describe above and that QAnon messaging exploits are resonant among a larger group of white, conservative audiences. Evidence from the 2016 US presidential election supports this broader appeal of a racial component among white conservatives, as prior work shows racial anxiety and resentment have been significant predictors of support for President Trump among this group (Riley and Peterson 2018; Reny, Collingwood, and Valenzuela 2019). More generally, research shows that, in America, people lack the same degree of empathy for children of color, reinforcing this racial component (Goff et al. 2014; Rattan et al. 2012). We therefore expect that QAnon's \#SaveTheChildren campaign will primarily depict risks to explicitly white children as a means to exploit these fears and anxieties and to gain more traction among these white, conservative audiences.

\subsection{Hypotheses and Predictions}

From this understanding of how charities and child-advocacy organizations depict children in their material, the mobilizing force of fear-evoking imagery on social media, and 
the exploitation of conservative audiences' white sexual politics, we develop several hypotheses about the imagery present in the QAnon co-opted campaign:

Hypothesis 1: QAnon depictions of children will skew heavily toward white youths. Building on the above central issues of white sexual politics, we anticipate that QAnon's attractiveness to the religious right and white, conservative voters will manifest as an overrepresentation of white children.

Hypothesis 2: QAnon will depict more female than male children. As suggested in BjorkJames 2020, protecting girls and women comprises a core tenet of white sexual politics and will result in a larger proportion of females in the QAnon dataset (BjorkJames 2020).

Hypothesis 3: QAnon depictions of children will contain a large incidence of graphic content. Following from the mobilizing effects of negative emotions and imagery, we anticipate QAnon imagery will largely include graphic, poverty porn-style depictions of children (i.e., children in distress, abused, in need, or in otherwise graphic situations) as a means to deliberately trigger emotional responses from the audience.

\section{Methodology}

\subsection{A Dataset of \#SaveTheChildren Images in Social Media}

Because social media platforms banned many QAnon-promoting accounts in the summer and fall of 2020 and winter of 2021, initial efforts to collect relevant images associated with QAnon's "Save the Children" or "Save our Children" campaigns have been challenging. Retrospective collection in Facebook and Instagram, enabled via CrowdTangle, was of limited success, as our collection efforts occurred after the platforms had already deleted or otherwise removed this content-outlined in Marshall 2020 as the "memory hole problem"-so we exclude this CrowdTangle data from our analysis.

While we have attempted to engage Facebook and access data removed as part of these platform moderation efforts, Facebook has not to date provided any of the requested data. Consequently, the research team turned to alternate online spaces for data collection, specifically Telegram, a semi-encrypted application popular among groups who have been banned from Facebook, Twitter, and mainstream spaces (Garry et al. 2021; Hoseini et al. 2021). As the QAnon campaign covers multiple platforms, and Facebook's deplatforming tactics do not directly suppress content shared on other platforms, we found QAnon accounts using the name "Save the Children" on Telegram. Likewise, prior work has shown that Telegram has become one of the major gathering points for QAnon content in the wake of interventions on mainstream platforms like Facebook, Instagram, and Twitter (Hoseini et al. 2021; Garry et al. 2021).

To collect images from Telegram, we relied on Telegram's support for searching based on hashtags. Querying for the hashtags \#SaveTheChildren and \#SaveOurChildren in May 2021 resulted in 300 unique groups that shared content with this hashtag. While many of these QAnon-associated accounts and groups (fraudulently) use the actual charity's red and white logo and name, the majority of groups on Telegram returned through this query were not associated with the charity (although there was one official account from 2018, which predates QAnon's co-option of the name). In these groups, we found thousands of images related to these queries. Many of these images were of women at demonstrations from all over the world that took place in the summer of 2020. As this work focuses on depictions of children in campaign imagery rather than photographic 


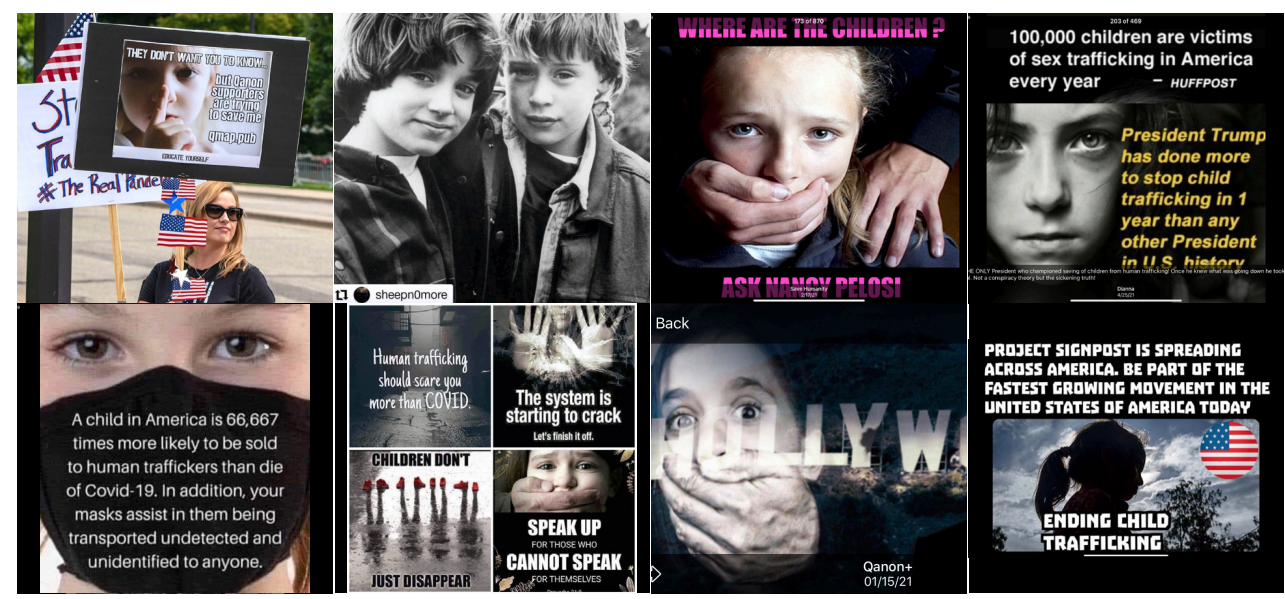

Figure 1: Sample of QAnon Images. People demonstrating (as in the top left) are common, as are images of celebrities (second image on the top left). Many images show children in distress or missing children.

coverage of people promoting the campaign at public rallies, we excluded these images of demonstrations and supporters - though this consistency suggests a broad support base from women. This filtering produced several hundred QAnon images, memes, and posters. To further expand potential sources for QAnon imagery, the research team also leveraged dozens of QAnon influencer accounts, including those of Lin Wood, Marjorie Taylor Greene, and Sidney Powell, to find images from the 2020 "Save the Children" campaign. After removing duplicates, this Telegram-focused collection contained 230 relevant and unique QAnon images (see Figure 1 for a sample of these images).

While our dataset does not include the date of the Telegram image posts, a subset of these images include information and captions describing when the image was posted or created. Of these 230 QAnon images, 50 include this time information, the majority of which were posted in May 2021 (19 images), followed by August 2020 (10 images). Of the remaining 21 images, 17 include dates between July 2020 and April 2021.

\subsection{Data on Actual Incidence of Child Trafficking}

As a point of reference and to compare depictions in QAnon images to real-world data on child trafficking, we rely on data from the Counter Trafficking Data Collaborative ${ }^{1}$ (CTDC). This organization provides global statistics on the incidence of child trafficking (including both sex- and labor-trafficking). Where possible, we also include data on US-specific child trafficking, as QAnon has its origins in the US. US data comes from the Polaris Project 2019 and the US National Human Trafficking Hotline. ${ }^{2}$

\subsection{Annotating Images for Age, Gender, Race, and Graphic Content}

To investigate QAnon's depictions of age, gender, race, and graphic content in its imagery, two members of the research team manually assessed each image for these categories; possible values for these codes are shown in Table 1 on the next page. We also coded each image's origin (e.g., Telegram, Twitter) and additional factors such as apparent geographic region of the image, child demeanor, and a description of the graphic content (if present). Where multiple children are depicted together in a single image, the annotation

1. https://www.ctdatacollaborative.org/

2. https://humantraffickinghotline.org/ 
guide instructed annotators to assess all children present (e.g., images with children of multiple ages or races should be labeled MIXED AGE and DIVERSE ETHNICITIES respectively).

Table 1: Annotation Options for \#SaveTheChildren Images

\begin{tabular}{llll} 
Age & Gender & Race & Graphic \\
\hline BABY & FEMALE & ASIAN & YES \\
TODDLER & MALE & BLACK/AFRICAN AMERICAN & NO \\
PRESCHOOLER & UNKNOWN & DIVERSE ETHNICITIES & \\
GRADE- & BOTH & HISPANIC/LATINX & \\
SCHOOLER & & & \\
TEEN & & INDIGENOUS & \\
YOUNG ADULT & & MIDDLE EASTERN & \\
MIXED AGE & & WHITE & \\
UNKNOWN & & UNKNOWN &
\end{tabular}

Describing Age in Depictions of Children To discretize ages of children in these images, we developed the following rules based on the AAP 2021 "Ages and Stages" guidelines:

- BABY: 0-12 months old

- TODDLER: $1-2$ years old

- PRESCHOOLER: $3-5$ years old

- GRADE-SCHOOLER: 6-12 years old

- TEEN: 13-17 years old

- YOUNG ADULT: 18-21 years old

Describing Gender The codebook includes labels for binary genders and supports instances where the annotator cannot tell the gender ("UNKNOWN") or children of multiple genders are present in the image ("BOTH"). We acknowledge, however, that a significant concern exists around the limited depiction of genderqueer youths in coding of visual media versus the disparate impact child trafficking has on that population. For this particular instance, where evidence suggests QAnon \#SaveTheChildren imagery primarily targets conservative voters, we expect overt instances of genderqueer depictions to be relatively rare. That said, incidence data from the US demonstrates genderqueer youths are disproportionately impacted by child trafficking, and yet it is difficult to identify genderqueer youths from imagery like that studied herein. As a result, our analysis may contribute to making an already disadvantaged and unseen population even more invisible. At the same time, trying to infer gender preferences from still images alone is also fraught with ethical concerns around agency and misidentification. Consequently, this work focuses on the presented genders of the children depicted. We understand and acknowledge this choice may be problematic.

Describing Race and Ethnicity Our definitions of race and ethnicity were also based largely on the descriptions provided by the US Census Bureau (Jensen et al. 2021). Our codebook augments these descriptions with a MIDDLE EASTERN category. 
As with annotating gender from static images, we acknowledge that imposing our own assessments of an individual's race and ethnicity is similarly ethically fraught-as with gender, research suggests individuals change their racial and ethnic identification over their lives-and introduces possible validity issues. For example, in Hannon and DeFina 2020 , results show significant disagreement in judging cross-race skin tone, and this disagreement persists even when the same annotator assesses the same image twice with two months between assessments. Difficulty in recognizing and differentiating among faces of individuals from races other than the annotator's own race is a wellknown cognitive bias, often referred to as the "own-race bias" (ORB) or "cross-race deficit" (CRD) effect, that is generally seen as "reliable across cultural and racial groups" (Meissner and Brigham 2001; Levin 2000). Consequently, identities and backgrounds of our annotators can potentially skew results, as this phenomenon suggests distinguishing among individuals of races apart from the annotator is a difficult task, as Hannon and DeFina 2020 show. We are certainly susceptible to this bias as well.

In the particular instance of this work, however, we potentially mitigate this validity issue-though we acknowledge it is not completely obviated. That is, the context of this work and the QAnon \#SaveTheChildren campaign focus primarily on depictions of white versus non-white children; while the CRD effect may impede our ability to separate children of two different races, as at least one author of this work identifies as white, studies of the ORB effect suggest an easier time is had separating members of one's own race from others (Ge et al. 2009). As a result, the bias here may confound results across race categorization, but white versus non-white distinctions are likely correct.

This potential mitigating factor aside, we continue to acknowledge potentially problematic aspects here. As with categorizations of gender, where genderqueer individuals experience an elevated risk of child trafficking, evidence suggests children of color likewise experience a disproportionate risk (US Department of State 2020; Davey 2020; Butler 2015). Therefore, misidentifying racial aspects of these populations potentially contributes to minimizing this disproportionate impact. Also as with gender, we anticipate imagery used by QAnon adherents in this campaign will primarily be comprised of white children, so this risk is somewhat reduced.

Describing Graphic Content To identify graphic content, annotators assessed whether the image portrayed nudity, blood, or bruising, or whether the child appeared scared and/or terrified. If the image implied violence to the child (sexual or otherwise), the image was coded as "graphic." In some cases, a child might appear sad or pensive, and the circumstances of their lives might include hardship, displacement, or malnutrition; however, these images were not coded as graphic, though if the child appeared sad or was not smiling, this was annotated. In cases where the children were portrayed in conflict settings, these images were not coded as graphic, although the context and location were noted.

\subsection{Assessing Agreement and Resolving Disagreement in Image Annotations}

Following initial annotation, annotators met to identify and discuss instances of disagreement across the image labels for age, gender, race, and graphic content. Initial assessments suggested strong agreement in the QAnon dataset across all four annotation tasks. Assessors identified several instances of disagreement in age and graphic content, which lead to clarifications on age ranges and on which instances of emotional state most closely aligned with the graphic content definition above. Despite this resolution discussion, some disagreements remained unresolved around race. 
In this manner, we resolved all instances of disagreement in three of the four primary factors. For remaining disagreements on race, we note the inter-annotator agreement using Krippendorff's $\alpha=0.8579$. Hence, annotator agreement in race is strong despite occasional disagreement. Where disagreement persisted across annotators, those instances were removed from downstream analysis.

\section{Results}

For each factor of interest, we first present descriptive statistics for label distributions to characterize QAnon images; for comparison, we have included statistics on actual child trafficking, where available or applicable. When calculating these metrics, we use only the images for which both annotators agree on a label, excluding labels of UNKNOWN.

\subsection{Depiction of Young Children in QAnon Images}

Focusing first on age, Tables 2 and 3 show the distributions of age assessments among QAnon images. Table 3 collapses ages into PRETEEN and TEEN groups, where TEEN includes both the TEEN and YOUNG ADULT categories. This assessment demonstrates that the majority of QAnon images depict preteen youths. Grade-school-aged children comprise the majority of images, while depictions of teenage youths and children under six are much rarer.

Comparing image data to global trafficking statistics for children under the age of 18 , as reported by the CTDC (third column in these tables, denoting a child's age when trafficking began), we see that depictions in the images do not reflect reality. These results suggest an overrepresentation of preteen youths in the QAnon dataset compared to global statistics. Restricting these statistics only to the US-as QAnon has its origins within the US-shows significant difference as well, with the most common age when trafficking began being 15-17 (47.93\%) (Polaris Project 2019).

Table 2: Distributions of Ages. Images primarily include grade-school-aged children, with teens being the second most common.

\begin{tabular}{lrr} 
Age & QAnon $(\mathrm{n}=230)$ & Globally \\
\hline BABY & $4.35 \%$ & $5.93 \%$ \\
TODDLER & $4.78 \%$ & $7.09 \%$ \\
PRESCHOOLER & $12.17 \%$ & $6.69 \%$ \\
GRADE-SCHOOLER & $60.43 \%$ & $29.29 \%$ \\
TEEN & $17.39 \%$ & $50.99 \%$ \\
YOUNG ADULT & $0.87 \%$ & - \\
MIXED AGE & $0 \%$ & -
\end{tabular}

Table 3: Distribution of Dichotomous Age Assessments. These images focus on depictions of preteen youths, clearly deviating from global statistics.

\begin{tabular}{lrr} 
Age, Binary & QAnon $(\mathrm{n}=230)$ & Globally \\
\hline PRETEEN & $81.74 \%$ & $49.01 \%$ \\
TEEN & $18.26 \%$ & $50.99 \%$
\end{tabular}




\subsection{Depictions of Gender in QAnon Images}

Moving to assessments of gender in depictions of children, Table 4 shows the breakdown of our results. Depictions of girls are much more common than boys in the QAnon images, with nearly three quarters of images including a girl.

Focusing only on depictions of either female or male children, we can compare incidence to global and US trafficking statistics (again, from the CTDC and Polaris Project, respectively). As shown in Table 5, results in QAnon images are broadly consistent with statistics from both the US and abroad, namely that girls are both more targeted and more often depicted in campaign material. Regarding Hypothesis 2, these results are consistent in that the QAnon imagery includes more depictions of female-presenting children.

Table 4: Distribution of Gender Assessments. After dropping images where the youths' presented gender was unknown, images are predominantly depictions of female children.

\begin{tabular}{lr} 
Gender & QAnon $(\mathrm{n}=217)$ \\
\hline FEMALE & $62.21 \%$ \\
MALE & $22.58 \%$ \\
BOTH & $15.21 \%$
\end{tabular}

Table 5: Distribution of Single-Gender Assessments. To compare against gender statistics in child trafficking, we find broad consistency among our datasets, where girls are more often targets of child trafficking than boys.

\begin{tabular}{lrrr} 
Gender & QAnon $(\mathrm{n}=184)$ & Globally & US \\
\hline FEMALE & $73.37 \%$ & $73.96 \%$ & $71.50 \%$ \\
MALE & $26.63 \%$ & $26.04 \%$ & $28.50 \%$
\end{tabular}

\subsection{Depictions of Race/Ethnicity in QAnon Images}

Table 6 presents the different races and ethnicities of children depicted in QAnon images in the dataset. It is immediately apparent that the QAnon images massively overrepresent white children, as four in every five images depicts such a child. While the CTDC data does not provide direct information about race and ethnicity of global trafficking survivors, the US-based National Center for Missing and Exploited Children has national statistics for this data. Also shown in Table 6, these statistics demonstrate that, even in the US, where white children do comprise a significant number of child-trafficking cases, children of color are disproportionately likely to be victims. This racial disparity in child trafficking is well-documented in the US and abroad by the US Department of State 2020 and other sources (e.g., Davey 2020; Butler 2015). These results and the QAnon imagery primarily depicting white youths are consistent with Hypothesis 1.

\subsection{Depiction of Graphic Content in QAnon Imagery}

As discussed above, recent marketing and outreach campaigns for charity organizations tend to avoid graphic, poverty-porn depictions of children. These organizations prefer instead to mobilize donors by sharing imagery of happy children, offering evidence of the positive impact that donations have on the children's lives. We therefore hypothesize that QAnon imagery would rely more on graphic depictions of children, exploiting negative emotions like fear and anger to mobilize their audience. 


\begin{abstract}
Table 6: Distribution of Race/Ethnicity Assessments. One can see the QAnon imagery predominantly contains depictions of white children. While white children account for the largest percentage in both the QAnon images and trafficked children in the US, they do not comprise the majority of trafficked children. Black/African American children are instead massively overrepresented in actual US trafficking statistics but appear only nine times in the QAnon dataset.
\end{abstract}

\begin{tabular}{lrr} 
Race & QAnon $(\mathrm{n}=213)$ & US \\
\hline ASIAN & $6.10 \%$ & $0.6 \%$ \\
BLACK/AFRICAN AMERICAN & $4.23 \%$ & $31 \%$ \\
DIVERSE ETHNICITIES & $4.23 \%$ & $10 \%$ \\
HISPANIC/LATINX & $2.35 \%$ & $18 \%$ \\
INDIGENOUS & $0 \%$ & $1.5 \%$ \\
MIDDLE EASTERN & $0 \%$ & - \\
WHITE & $83.10 \%$ & $36 \%$
\end{tabular}

Table 7 presents the prevalence of graphic depictions in the QAnon dataset, showing that, indeed, QAnon content contains many graphic depictions. While the overall rate of graphic depictions in the QAnon data is lower than one might expect, only accounting for about one in three images, these results are broadly consistent with Hypothesis 3, as we would expect a modern charity campaign to use such depictions only sparingly (Lentfer 2018).

Table 7: Distribution of Graphic Content Assessments. QAnon imagery, as expected, contains a sizable proportion of graphic depictions.

\begin{tabular}{lr} 
Graphic Content & QAnon $(\mathrm{n}=230)$ \\
\hline YES & $35.22 \%$ \\
NO & $64.78 \%$
\end{tabular}

\title{
4.5 Post Hoc Analysis: Race in Recurring Visual Tropes
}

Following assessment of the QAnon imagery, we identified a recurring depiction of a child being silenced by an abusive or threatening hand. Of the 230 QAnon images in the dataset, we identified this visual trope in 18 images, and of these, 12 included depictions of darker-skinned abusers. The majority of these 18 images show young, preteen girls. Figure 2 on the following page shows a sample of these images.

\section{Discussion}

QAnon \#SaveTheChildren imagery tends to depict young, white, female children, often in graphic distress or violent situations. Though these images depart from the realities of child trafficking, they appear founded in the familiar but outmoded poverty-porn tropes of children in distress and a focus on female children. As discussed in O'Dell 2008 and in Wymer and Gross 2021, historical charity campaigns have used similar depictions of children in distress-especially young girls-and have included visuals similar to those in our post hoc analysis-an older male, often in a threatening position, touching the face of the victimized child. QAnon's use of gender is also similar to real-world statistics, 


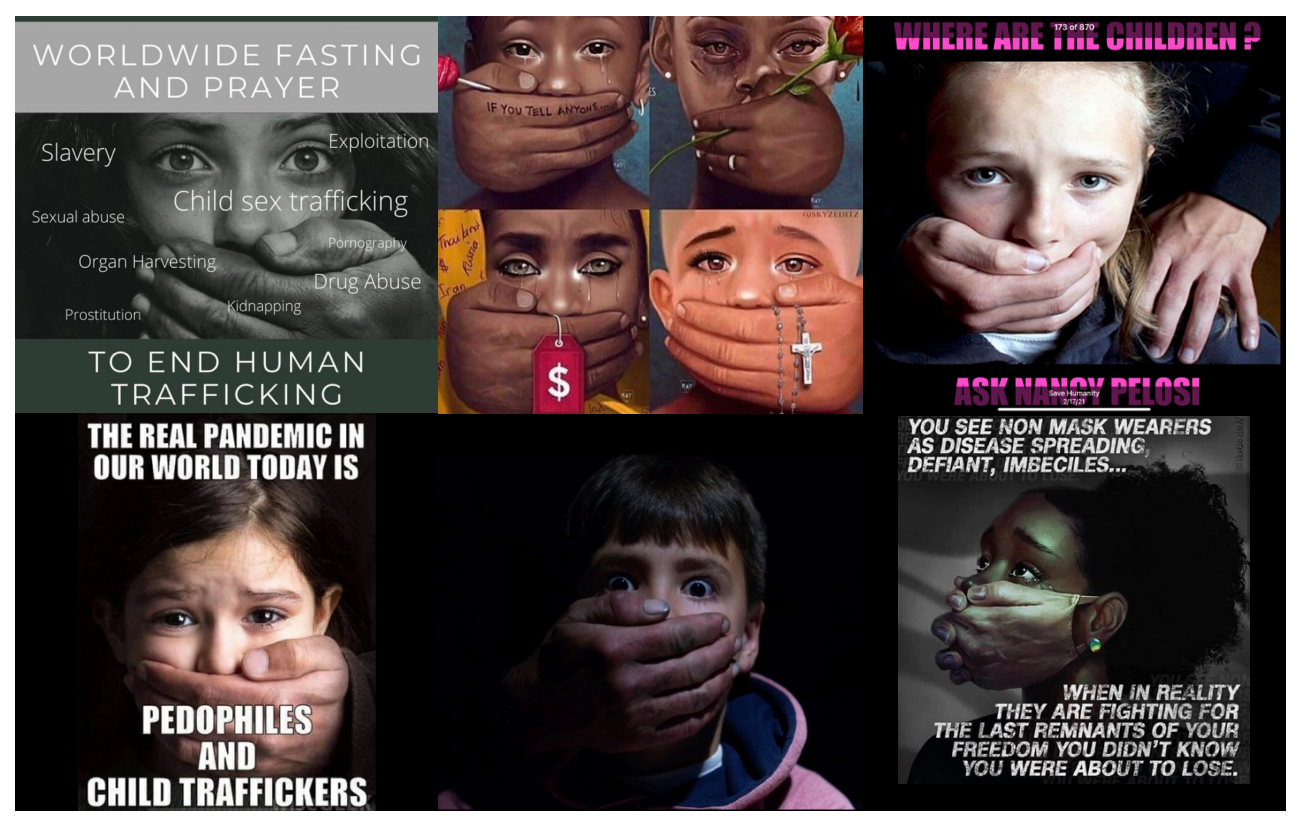

Figure 2: A Sample of a Recurring Racialized Trope. The QAnon dataset contains 18 examples of this threatening trope of a child with a menacing hand touching them or covering their mouths. Of these 18 images, 12 appear to have darker-skinned abusers.

where the preponderance of girls in QAnon images is similar to statistics in the US and globally. ${ }^{3}$

The QAnon campaign then departs from this familiar motif in a warped depiction of child trafficking in both age and race: QAnon imagery depicts more young children than actual statistics would suggest and diverges massively in victims' apparent races, greatly overrepresenting white children compared to other races and ethnicities. Four of every five images in the QAnon dataset depict a white child, making white children far and away the most commonly depicted race in the set. Even if we restrict ourselves to childtrafficking statistics in the US, where white children are more likely to be the victims of child trafficking, QAnon imagery still massively underrepresents the incidence of Black children.

This unrealistic depiction of white children is consistent with an effort to exploit the concerns in white sexual politics as outlined in Factor 3. That is, these depictions may be particularly activating for white, conservative audiences given their concerns around protecting white privilege, hetero-/patriarchal norms, and their children and families from an "other" out-group. While the out-group threatening their children may be fluide.g., darker-skinned individuals as shown in Figure 2, liberal elites as in the original pedophilic-cabal conspiracy, or members of the LGBTQ+ community (Figures 3a and 3b) -a consistent narrative in the imagery used in QAnon's \#SaveTheChildren campaign is that white children are in danger and need protection.

This narrative's intersection with the anxieties of white, conservative audiences and graphic depictions of children suggests these audiences are likely to have strong and negative reactions to these images. As Dahmen, Mielczarek, and Morrison 2019 note, the emotional impact of such distressing images is well-known, and "such affective reactions happen on a neural level." Consequently, exposure to these images may drive additional

3. We note here that these results invisiblize genderqueer children, which reports show have higher rates of victimization, as we discuss in the Describing Gender section. 


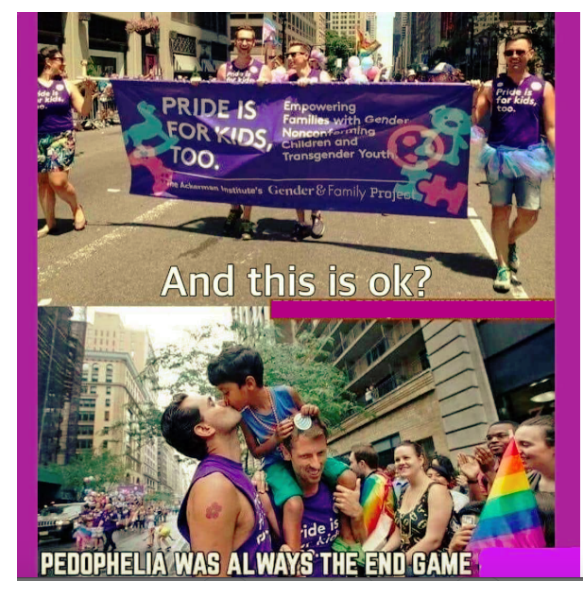

(a) Channeling fear of LGBTQ+ groups, this image casts those violating heterosexual norms as pedophilic "others."

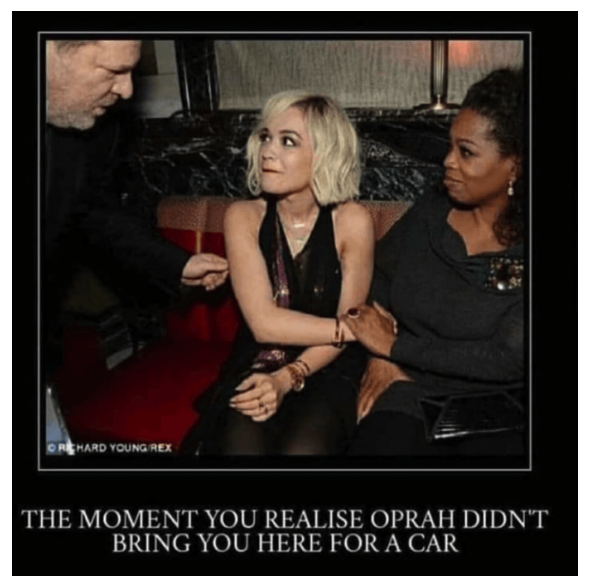

(b) Highlighting young, white women as victims, this image suggests celebrities and those of color are abusers.

Figure 3: Images from QAnon's \#SaveTheChildren Campaign. These images show how children and young white women are portrayed as in need of protection.

mobilization and propensity to share these messages among these audiences, as Factor 2 and Casas and Williams 2019 suggest.

\subsection{Interventions Against Malevolent Appropriation}

Identifying these three factors-appropriating a familiar visual motif, triggering fear and anger with graphic images of this motif, and exploiting racially driven fears in white audiences-yields insight into potential interventions. Regarding appropriation, realworld events, social ills, and contentious topics are often fodder for exploitation by malevolent groups-e.g., influence campaigns' exploitation of civil rights issues and Black Lives Matter (Arif, Stewart, and Starbird 2018). Such risks are so prevalent on social media that a term for this appropriation exists in public-relations literature: "hatejacking" (Benton and Peterka-Benton 2020). To combat hatejacking, Benton and Peterka-Benton 2020 outline how organizations' disavowal of connections with hate groups can backfire and provide more visibility and social capital to these groups. Hence, insights from Benton and Peterka-Benton 2020 suggest that legitimate organizations whose campaigns are appropriated in this way should halt their campaigns and not respond, thereby reducing opportunity for further exposure. These responses are definitionally reactive, however, and are useful only after a campaign of appropriation has begun.

Regarding the use of fear- and anger-evoking images to stimulate mobilization and sharing, as discussed in Factor 2, online platforms and technology companies may have opportunities for proactive interventions. The graphic nature of many images used by the QAnon \#SaveTheChildren campaign potentially separates them from those used by modern charities, as contemporary campaigns are often more positive in their depictions (Wymer and Gross 2021; O'Dell 2008; Rijn, Barham, and Sundaram-Stukel 2017). This differentiation may open avenues for intervention, as our ability to automatically characterize images (Joo and Steinert-Threlkeld 2018) and recognize hateful content therein improves (Kiela et al. 2021); the platforms can then develop methods to suppress images that evoke fear or anger or present similar exploitative imagery. Platforms like YouTube have demonstrated success in modifying their recommendation systems to suppress similar kinds of alternative content, which has had downstream effects on the sharing of such content in other spaces (Buntain et al. 2021). While such suppressive efforts are fraught with questions of censorship, these approaches could limit the use 
of graphic, negative content for manipulation while allowing pro-social use of positive imagery.

\subsection{Possibilities for Future Appropriation}

QAnon imagery also includes recognizable images of child-actors such as Heather O’Rourke, Macaulay Culkin, and Drew Barrymore. Given sexual harassment and assault in the entertainment industry exposed as part of the \#MeToo movement, use of these images is concerning as they might lend legitimacy to conspiracy claims. These abuses (especially among child actors) may provide legitimacy for QAnon conspiracies around sexual predation by liberal/celebrity elites while also setting up a new opportunity for appropriation. Though the \#MeToo movement may be more difficult to appropriate as it lacks a similarly consistent visual motif, images such as Figure $3 \mathrm{~b}$ demonstrate a potential link. This image may similarly evoke emotions of fear and anger; it also exploits racial drivers, where a white young woman is supposedly being offered to a predator by a Black person. Hence, the second and third factors we mention suggest a space exists for a similar risk of appropriation. Given the \#MeToo movement's connection to liberal audiences, such appropriation may also represent a vector for exposing more liberal audiences to QAnon messaging.

\section{Threats to Validity}

A core possible threat to the validity of this work comes from the difficulty in clearly attributing content to the QAnon movement versus one of the other groups in the ecology of extremist and/or hate organizations. For example, non-trivial overlap exists between white supremacist groups and QAnon adherents (Garry et al. 2021), making it possible that some images posted with \#SaveTheChildren and related hashtags may not have come directly from a QAnon adherent. This threat complicates clear attribution and strategy to a particular group. That said, however, as we mention when we first outline the three factors providing space for this campaign's success, it is likely incorrect to ascribe a single overarching and coherent strategy to the QAnon \#SaveTheChildren campaign. Rather, in this work, we are more interested in the visual depictions to which an audience member-QAnon adherent or not-might be exposed, as this work is about understanding the context and potential factors driving engagement with this campaign. QAnon messaging exists in a complex ecosystem of mainstream and extreme groups and fringe and major platforms, suggesting many individuals may be working together to advance this appropriated \#SaveTheChildren campaign. Either way, this co-opted campaign's success is difficult to dispute, and the factors driving its exposure to new audiences are important to understand if we want to make online spaces safer from this sort of exposure and appropriation.

\section{Conclusions}

The QAnon movement's ability to parlay societal ills into vectors for reaching new audiences-as in the QAnon \#SaveTheChildren campaign-threatens the safety of online audiences and has directly impacted child-advocacy groups' abilities to support children. The factors contributing to QAnon's success in this audience-building and recruitment remain unclear, however, which impedes development of effective defenses, interventions, and counter-messaging. By posing three factors that may have contributed to the success of QAnon's \#SaveTheChildren campaign-the appropriation of a familiar visual motif around child suffering that anchors the campaign's imagery; the mobilizing 
power of images laden with negative emotions that may stimulate response; and the exploitation of concerns among white, conservative audiences by depicting threats to white children-we hope to establish a foundation for developing these counters and making the online information ecosystem a safer place.

To this end, we have characterized the imagery used in QAnon's campaign, finding that these images depict a consistent theme of exploited young white girls, often shown in graphic situations. This theme deviates from the reality of child trafficking in age and race, as young, white children in need are overrepresented in QAnon imagery compared to realworld statistics. Using these characterizations and their connections to the three outlined factors, we then outline potential interventions for hindering similar malevolent, imageoriented campaigns and identify a topic that may be at risk for similar appropriation: the \#MeToo movement. Given the QAnon movement's continued reach and connection to violence, more work is needed to evaluate these interventions and address these risks. 


\section{References}

American Academy of Pediatrics. 2021. "Ages and Stages.” Accessed September 27, 2021. https://www.healthychildren.org/English/ages-stages/Pages/default.aspx.

Argentino, Marc-Andre, and Blyth Crawford. 2021. "The WQmen of QAnon.” Global Network on Extremism and Technology (March). https://gnet-research.org/2021/ 03/12/the-wqmen-of-qanon/.

Arif, Ahmer, John J Robinson, Stephanie A Stanek, Elodie Fichet, Paul Townsend, Zena Worku, and Kate Starbird. 2017. "A Closer Look at the Self-Correcting Crowd: Examining Corrections in Online Rumors.” CSCW, https://doi.org/10.1145/2998181. 2998294.

Arif, Ahmer, Leo Graiden Stewart, and Kate Starbird. 2018. "Acting the Part: Examining Information Operations Within \#BlackLivesMatter Discourse." Proc. ACM Hum.Comput. Interact. (New York, NY, USA) 2, no. CSCW (November): 20:1-20:27. https: //doi.org/10.1145/3274289.

Auxier, Brooke. 2020. "64\% of Americans say social media have a mostly negative effect on the way things are going in the U.S. today." Pew Research Center (October). https://www.pewresearch.org/fact-tank/2020/10/15/64-of-americans-say-socialmedia-have-a-mostly-negative-effect-on-the-way-things-are-going-in-the-u-stoday/.

Benton, Bond, and Daniela Peterka-Benton. 2020. "Hating in plain sight: The hatejacking of brands by extremist groups." Public Relations Inquiry 9 (1): 7-26. https://doi.org/ 10.1177/2046147X19863838.

Berger, Jonah, and Katherine L. Milkman. 2012. "What makes online content viral?” Journal of Marketing Research 49 (2): 192-205. https://doi.org/10.1509/jmr.10. 0353.

Bigio, Jamille, and Elena Ortiz. 2021. "The Threat of Human Trafficking to National Security, Economic Growth, and Sustainable Development.” Council on Foreign Relations (February). https://www.cfr.org/blog/threat-human-trafficking-national-securityeconomic-growth-and-sustainable-development.

Bjork-James, Sophie. 2020. "White Sexual Politics: The Patriarchal Family in White Nationalism and the Religious Right." Transforming Anthropology 28 (1): 58-73. https://doi.org/10.1111/traa.12167.

Bloom, Mia, and Sophia Moskalenko. 2021. Pastels and Pedophiles: Inside the Mind of QAnon. Stanford, CA: Redwood/Stanford University Press.

Bonaparte, Yvette Lynne. 2020. “Meeting the Moment: Black Lives Matter, Racial Inequality, Corporate Messaging, and Rebranding." Advertising \& Society Quarterly 21 (3). https://doi.org/10.1353/asr.2021.0000.

Borah, Abhishek, Sourindra Banerjee, Yu Ting Lin, Apurv Jain, and Andreas B. Eisingerich. 2020. "Improvised Marketing Interventions in Social Media." Journal of Marketing 84 (2): 69-91. https://doi.org/10.1177/0022242919899383.

Bracewell, Lorna. 2021. "Gender, Populism, and the QAnon Conspiracy Movement." Frontiers in Sociology 5 (January): 3-6. https://doi.org/10.3389/fsoc.2020.615727.

Brantly, Aaron Franklin. 2019. "From Cyberspace to Independence Square: Understanding the Impact of Social Media on Physical Protest Mobilization During Ukraine's Euromaidan Revolution.” Journal of Information Technology and Politics 16 (4): 36078. https://doi.org/10.1080/19331681.2019.1657047. 
Buntain, Cody, Richard Bonneau, Jonathan Nagler, and Joshua A Tucker. 2021. "YouTube recommendations and effects on sharing across online social platforms." PACM on Human-Computer Interaction, https:// doi .org/10 .1145/3449085. eprint: 2003.00970.

Butler, Cheryl Nelson. 2015. "The racial roots of human trafficking." UCLA Law Review 62 (6): 1464-514. https://www.uclalawreview.org/racial-roots-human-trafficking/.

Casas, Andreu, and Nora Webb Williams. 2019. "Images that Matter: Online Protests and the Mobilizing Role of Pictures." Political Research Quarterly 72 (2): 360-75. https://doi.org/10.1177/1065912918786805.

Chafkin, Max. 2021. “Peter Thiel’s Origin Story.” New York Magazine (September). https: // nymag.com/intelligencer/article/ peter-thiel-silicon-valley-contrarian-maxchafkin.html.

Civiqs. 2020. National Politics Survey September 2020. Technical report September. Civiqs Daily Kos. https://civiqs.com/documents/Civiqs_DailyKos_monthly_banner_ book_2020_09_klw74f.pdf.

Conger, Kate. 2020. “Twitter Takedown Targets QAnon Accounts.” New York Times (New York) (July). https://www.nytimes.com/2020/07/21/technology/twitter-bansqanon-accounts.html.

_. 2021. "Twitter, in Widening Crackdown, Removes Over 70,000 QAnon Accounts." New York Times (New York) (January). https://www.nytimes.com/2021/01/11/ technology/twitter-removes-70000-qanon-accounts.html.

Dahmen, Nicole Smith, Natalia Mielczarek, and Daniel D Morrison. 2019. "The (in)disputable 'power' of images of outrage: public acknowledgement, emotional reaction, and image recognition." Visual Communication 18 (4): 453-74. https://doi.org/10. $1177 / 1470357217749999$.

Davey, Samantha. 2020. Snapshot on the State of Black Women and Girls: Sex Trafficking in the U.S. Technical report. Congressional Black Caucus Foundation. https://doi. org/10.4324/9780203504307.

Dickinson, Ej. 2020. “'Pastel QAnon' Is Infiltrating the Natural Parenting Community.” Rolling Stone (December). https://www.rollingstone.com/culture/culture-news/ qanon-pastel-antivax-natural-parenting-community-freebirth-1098518/.

Enjolras, Bernard, Kari Steen-Johnsen, and Dag Wollebæk. 2013. "Social media and mobilization to offline demonstrations: Transcending participatory divides?" New Media and Society 15 (6): 890-908. https://doi.org/10.1177/1461444812462844.

Facebook. 2021. An Update to How We Address Movements and Organizations Tied to Violence. Accessed September 20, 2021. https://about.fb.com/news/2020/08/ addressing-movements-and-organizations-tied-to-violence/.

Fan, Rui, Jichang Zhao, Yan Chen, and Ke Xu. 2014. "Anger is more influential than joy: Sentiment correlation in Weibo.” PLoS ONE 9 (10). https://doi.org/10.1371/journal. pone.0110184. arXiv: 1309.2402.

Forrest, Brett. 2021. "What Is QAnon? What We Know About the Conspiracy-Theory Group.” Wall Street Journal (February). https://www.wsj.com/articles/what-isqanon-what-we-know-about-the-conspiracy-theory-11597694801. 
Freelon, Deen, Michael Bossetta, Chris Wells, Josephine Lukito, Yiping Xia, and Kirsten Adams. 2020. "Black Trolls Matter: Racial and Ideological Asymmetries in Social Media Disinformation.” Social Science Computer Review, 1-19. https://doi.org/10. 1177/0894439320914853.

Garry, Amanda, Samantha Walther, Rukaya Mohamed, and Ayan Mohammed. 2021. "QAnon Conspiracy Theory: Examining its Evolution and Mechanisms of Radicalization.” Journal for Deradicalization Spring (26): 152-216. https://journals.sfu.ca/jd/ index.php/jd/article/view/437/265.

Ge, Liezhong, Hongchuan Zhang, Zhe Wang, Paul C Quinn, Olivier Pascalis, David Kelly, Alan Slater, Jie Tian, and Kang Lee. 2009. "Two faces of the other-race effect: Recognition and categorisation of Caucasian and Chinese faces." Perception 38 (8): 1199210. https://doi.org/10.1068/p6136.

Goff, Phillip Atiba, Matthew Christian Jackson, Brooke Allison Lewis Di Leone, Carmen Marie Culotta, and Natalie Ann DiTomasso. 2014. "The essence of innocence: Consequences of dehumanizing black children." Journal of Personality and Social Psychology 106 (4): 526-45. https://doi.org/10.1037/a0035663.

Guo, Chao, and Gregory D. Saxton. 2018. "Speaking and Being Heard: How Nonprofit Advocacy Organizations Gain Attention on Social Media." Nonprofit and Voluntary Sector Quarterly 47 (1): 5-26. https://doi.org/10.1177/0899764017713724.

Hannah, Matthew. 2021. "QAnon and the information dark age." First Monday 26 (2). https://doi.org/10.5210/fm.v26i2.10868.

Hannon, Lance, and Robert DeFina. 2020. "The Reliability of Same-Race and Cross-Race Skin Tone Judgments.” Race and Social Problems 12 (2): 186-94. https://doi.org/ 10.1007/s12552-020-09282-4.

Heffernan, Virginia. 2020. “QAnon's monstrous conspiracy theories fit the Trumpian moment.” Los Angeles Times (Los Angeles) (September). https://www.latimes.com/ opinion/story/2020-09-18/qanon-tom-hanks-blood-libel-protocols-of-the-elders.

Hoseini, Mohamad, Philipe Melo, Fabricio Benevenuto, Anja Feldmann, and Savvas Zannettou. 2021. "On the Globalization of the QAnon Conspiracy Theory Through Telegram.” In arXiv preprint. https:// doi .org/10.48550/arXiv.2105.13020. arXiv: 2105.13020.

Howard, Philip N, Aiden Duffy, Deen Freelon, Muzammil M Hussain, Will Mari, and Mrawa Marwa Mazaid. 2011. "Opening closed regimes: what was the role of social media during the Arab Spring?," 1-30. https://doi.org/10.1007/s13398-014-0173-7.2. http://papers.ssrn.com/sol3/papers.cfm?abstract_id=2595096.

Jensen, Eric, Nicholas Jones, Kimberly Orozco, Lauren Medina, Marc Perry, Ben Bolender, and Karen Battle. 2021. "Measuring Racial and Ethnic Diversity for the 2020 Census." Accessed September 27, 2021. https://www.census.gov/newsroom/blogs/randomsamplings/2021/08/measuring-racial-ethnic-diversity-2020-census.html.

Joo, Jungseock, and Zachary C. Steinert-Threlkeld. 2018. "Image as Data: Automated Visual Content Analysis for Political Science,” 1-38. eprint: 1810.01544. http: //arxiv.org/abs/1810.01544.

Kelly, Annie. 2020. “Mothers for QAnon.” New York Times (New York) (September). https: //www.nytimes.com/2020/09/10/opinion/qanon-women-conspiracy.html. 
Kiela, Douwe, Hamed Firooz, Aravind Mohan, Vedanuj Goswami, Amanpreet Singh, Casey A Fitzpatrick, Peter Bull, Greg Lipstein, Tony Nelli, Ron Zhu, et al. 2021. "The Hateful Memes Challenge : Competition Report." In NeurIPS 2020 Competition and Demonstration Track, 344-60. Proceedings of Machine Learning Research. http: //proceedings.mlr.press/v133/kiela21a/kiela21a.pdf.

Lentfer, Jennifer. 2018. "Yes, charities want to make an impact. But poverty porn is not the way to do it." The Guardian (January).

Levin, Daniel T. 2000. "Race as a visual feature: Using visual search and perceptual discrimination tasks to understand face categories and the cross-race recognition deficit." Journal of Experimental Psychology: General 129 (4): 559-74. https://doi. org/10.1037/0096-3445.129.4.559.

Lilleker, Darren G., Anastasia Veneti, and Daniel Jackson. 2019. "Introduction: Visual Political Communication." In Visual Political Communication, edited by Anastasia Veneti, Daniel Jackson, and Darren G Lilleker, 1-13. Cham: Springer International Publishing. https://doi.org/10.1007/978-3-030-18729-3_1.

Lovejoy, Kristen, and Gregory D. Saxton. 2012. "Information, Community, and Action: How Nonprofit Organizations Use Social Media.” Journal of Computer-Mediated Communication 17 (3): 337-53. https://doi.org/10.1111/j.1083-6101.2012.01576. $\mathrm{x}$.

Marshall, John McClellan. 2020. "The Modern Memory Hole: Cyberethics Unchained.” Athenaeum Review 1 (3). https://athenaeumreview.org/essay/the-modern-memoryhole-cyberethics-unchained/.

McNeal, Stephanie. 2020. "The Conspiracy Theory About Wayfair Is Spreading Fast Among Lifestyle Influencers On Instagram." BuzzFeed News (July). https://www.bu zzfeednews.com/article/stephaniemcneal/wayfair-qanon-influencers-instagram.

Meissner, Christian A, and John C Brigham. 2001. "Thirty Years of Investigating the Own-Race Bias in Memory for Faces: A Meta-Analytic Review." Psychology, Public Policy, and Law 7 (1): 3-35. https://doi.org/10.1037/1076-8971.7.1.3.

Moskalenko, Sophia, and Clark McCauley. 2021. "QAnon: Radical Opinion versus Radical Action." Perspectives on Terrorism 15 (2): 142-46.

O'Dell, Lindsay. 2008. “Representations of the 'damaged' child: 'Child saving' in a British children's charity ad campaign." Children and Society 22 (5): 383-92. https://doi. org/10.1111/j.1099-0860.2007.00114.x.

Papasavva, Antonis, Jeremy Blackburn, Gianluca Stringhini, Savvas Zannettou, and Emiliano De Cristofaro. 2021. ““Is it a Qoincidence?”: An Exploratory Study of QAnon on Voat." The Web Conference 2021 - Proceedings of the World Wide Web Conference, WWW 2021, no. August, 460-71. https://doi.org/10.1145/3442381.3450036. arXiv: 2009.04885.

Petrosky, Miguel. 2020. “Christian QAnon'ers Want To \#SaveTheChildren. They're Making Things Worse,” October. https : / / sojo . net / articles / christian - qanoners - want savethechildren-theyre-making-things-worse.

Polaris Project. 2019. 2018 Statistics from the National Human Trafficking Hotline. Technical report. Polaris Project. https://polarisproject.org/wp-content/uploads/2019/ 09/Polaris_National_Hotline_2018_Statistics_Fact_Sheet.pdf. 
Rajan, Anjana, Catherine Chen, Benjamin Caren, Mollie Saltskog, Jason Blazakis, Zach Schwitzky, and Leela McClintock. 2021. Countering QAnon. Technical report February. Polaris Project. https://polarisproject.org/wp-content/uploads/2021/02/PolarisReport-Countering-QAnon.pdf.

Rathje, Steve, Jay J. Van Bavel, and Sander van der Linden. 2021. "Out-group animosity drives engagement on social media." Proceedings of the National Academy of Sciences 118 (26): e2024292118. https://doi.org/10.1073/pnas.2024292118.

Rattan, Aneeta, Cynthia S. Levine, Carol S. Dweck, and Jennifer L. Eberhardt. 2012. "Race and the fragility of the legal distinction between juveniles and adults." PLOS ONE 7 (5): 5-9. https://doi.org/10.1371/journal.pone.0036680.

Reny, Tyler T, Loren Collingwood, and Ali A Valenzuela. 2019. "Vote switching in the 2016 election: How racial and immigration attitudes, not economics, explain shifts in white voting." Public Opinion Quarterly 83 (1): 91-113. https://doi.org/10.1093/ poq/nfz011.

Rijn, Jordan van, Bradford Barham, and Reka Sundaram-Stukel. 2017. "An experimental approach to comparing similarity- and guilt-based charitable appeals." Journal of Behavioral and Experimental Economics 68:25-40. https://doi.org/10.1016/j.socec. 2017.02.004.

Riley, Emmitt Y, and Clarissa Peterson. 2018. “Economic Anxiety or Racial Predispositions? Explaining White Support for Donald Trump in the 2016 Presidential Election." Journal of Race and Policy 14 (1): 5-24. https://doi.org/10.2139/ssrn.2847791.

Rogers, Kaleigh. 2020. “Trump Said QAnon ‘Fights’ Pedophilia. But The Group Has Made It Harder To Protect Kids." FiveThirtyEight.com (October). https:// fivethirtyeight. com/features/qanons-obsession-with-savethechildren-is-making-it-harder-tosave-kids-from-traffickers/.

Roose, Kevin. 2019. “Why Banning 8chan Was So Hard for Cloudflare: 'No One Should Have That Power'." New York Times (New York) (August). https://www.nytimes.com/ 2019/08/05/technology/8chan-cloudflare-el-paso.html.

_ 2020a. “How ‘Save the Children’ Is Keeping QAnon Alive.” New York Times (New York) (September). https://www.nytimes.com/2020/09/28/technology/save-thechildren-qanon.html.

- 2020b. "QAnon Followers Are Hijacking the \#SaveTheChildren Movement." New York Times (August). https://www.nytimes.com/2020/08/12/technology/qanonsave-the-children-trafficking.html.

.2021. “A QAnon ‘Digital Soldier’ Marches On, Undeterred by Theory’s Unraveling.” New York Times (January). https: / / www . theguardian . com / voluntary - sector network/2018/jan/12/charities-stop-poverty-porn-fundraising-ed-sheeran-comicrelief.

Sánchez-Cuenca, Ignacio, and Paloma Aguilar. 2009. "Terrorist violence and popular mobilization: The case of the Spanish transition to democracy." Politics and Society 37 (3): 428-53. https://doi.org/10.1177/0032329209338927.

Save the Children. 2020. Save the Children Statement on use of its Name in Unaffiliated Campaigns, August. Accessed February 11, 2022. https://www.savethechildren. org/us/about-us/media-and-news/2020-press-releases/save-the-childrenstatement-on-use-of-its-name-in-unaffiliated-c. 
Seitz, Amanda. 2020. “QAnon's 'Save the Children' morphs into popular slogan.” AP News (October). https://apnews.com/article/election-2020-donald-trump-childtrafficking-illinois-morris-aab978bb7e9b89cd2cea151ca13421a0.

Seo, Hyunjin. 2014. "Visual Propaganda in the Age of Social Media: An Empirical Analysis of Twitter Images During the 2012 Israeli-Hamas Conflict." Visual Communication Quarterly 21 (3): 150-61. https://doi.org/10.1080/15551393.2014.955501.

Shaer, Matthew. 2014. "What Emotion Goes Viral the Fastest? On Twitter and Facebook, which spreads quickest: joy, sadness or disgust?” Smithsonian Magazine (April). https://www.smithsonianmag.com/science-nature/what-emotion-goes-viralfastest-180950182/.

UNODC. 2020. Global Report on Trafficking in Persons 2020. Technical report. UNODC. https://doi.org/10.1007/978-3-319-74336-3_221-1. https://www.unodc.org/ documents/data-and-analysis/tip/2021/GLOTiP_2020_15jan_web.pdf.

US Department of State. 2020. Trafficking In Persons Report. Technical report. Washington, DC: Department of State. https://www.state.gov/reports/2020-trafficking-inpersons-report/.

Vosoughi, Soroush, Deb Roy, and Sinan Aral. 2018. "The spread of true and false news online.” Science 359 (March): 1146-51. https://doi.org/10.1126/science.aap9559.

Vrzal, Miroslav. 2020. "QAnon as a variation of a Satanic conspiracy theory : an overview." Theory and Practice in English Studies 9 (1). http://hdl.handle.net/11222.digilib/ 143485.

Wilson, Tom, Kaitlyn Zhou, and Kate Starbird. 2018. "Assembling strategic narratives: Information operations as collaborative work within an online community." Proceedings of the ACM on Human-Computer Interaction 2 (CSCW). https://doi.org/10.1145/ 3274452.

Wymer, Walter, and Hellen Gross. 2021. "Charity advertising: A literature review and research agenda." Journal of Philanthropy and Marketing n/a (n/a): e1723. https: //doi.org/10.1002/nvsm.1723.

Yamamoto, Masahiro, Matthew J. Kushin, and Francis Dalisay. 2015. "Social media and mobiles as political mobilization forces for young adults: Examining the moderating role of online political expression in political participation." New Media and Society 17 (6): 880-98. https://doi.org/10.1177/1461444813518390.

Yarlott, W. Victor H., and Mark A. Finlayson. 2016. "Learning a Better Motif Index: Toward Automated Motif Extraction." In 7th Workshop on Computational Models of Narrative (CMN 2016), edited by Ben Miller, Antonio Lieto, Rémi Ronfard, Stephen G. Ware, and Mark A. Finlayson, vol. 53, 7:1-7:10. OpenAccess Series in Informatics (OASIcs). Dagstuhl, Germany: Schloss Dagstuhl-Leibniz-Zentrum fuer Informatik. https://doi. org/10.4230/OASIcs.CMN.2016.7.

Yonetani, Ryo, Kris M Kitani, and Yoichi Sato. 2016. "Visual Motif Discovery via FirstPerson Vision." In Computer Vision - ECCV 2016, edited by Bastian Leibe, Jiri Matas, Nicu Sebe, and Max Welling, 187-203. Cham: Springer International Publishing.

Zannettou, Savvas, Tristan Caulfield, Barry Bradlyn, Emiliano De Cristofaro, Gianluca Stringhini, and Jeremy Blackburn. 2020. "Characterizing the Use of Images in StateSponsored Information Warfare Operations by Russian Trolls on Twitter." Proceedings of the International AAAI Conference on Web and Social Media 14, no. 1 (May): 774-85. eprint: 1901.05997. https://ojs.aaai.org/index.php/ICWSM/article/view/ $7342 / 7196$. 
Zielinski, Graeme, and David Jackson. 1998. "At Times, I've Wanted To Turn It Off Too.” Chicago Tribune (March). https://www.chicagotribune.com/news/ct-xpm-1998-0315-9803150059-story.html.

Zuckerman, Ethan. 2019. "QAnon and the Emergence of the Unreal." Journal of Design and Science, no. 6, https://doi.org/10.21428/7808da6b.6b8a82b9. 


\section{Authors}

Cody Buntain is an assistant professor at the University of Maryland, College Park cbuntain@umd.edu.

Monique Deal Barlow is a doctoral candidate in the Political Science Department at Georgia State University in Atlanta, Georgia.

Mia Bloom is a Professor of Communication at Georgia State University in Atlanta, Georgia.

Mila A. Johns is an independent researcher/consultant based in Washington, DC.

\section{Acknowledgements}

We are grateful to Dr. Holly Pinheiro, who provided feedback on an earlier draft.

\section{Data Availability Statement}

Data that supports the findings of this study are available upon request from the corresponding author, Cody Buntain. This data is not publicly available owing to privacy concerns for those depicted in the QAnon imagery.

\section{Funding Statement}

The research was supported by the Minerva Research Initiative, Department of Defense Grant \#N000 14-21-275485. Any opinions, findings, or recommendations expressed in this article are those of the authors alone and do not reflect the views of the Office of Naval Research, the Department of the Navy, or the Department of Defense.

\section{Conflict of Interest}

Not applicable.

\section{Ethical Standards}

The Minerva project \#N000 14-21-275485 for which this article was supported received an IRB exemption from Georgia State University, H21396. Reference Number: 366603.

A considerable ethical issue emerging in this research concerns the depiction of children in these images. Children, especially those in need and/or already exploited, are already an at-risk population, and the QAnon imagery used for this analysis further exploits them. Especially for children who were actually reported as missing, including their depictions in the dataset is ethically fraught: On the one hand, we are discussing an existing exploitative narrative, but on the other, this work uses and therefore propagates and benefits from these depictions. While a solution could be to anonymize images in this dataset, such obfuscation would significantly impact replication of our analysis, as assessing gender, age, and ethnicity are already sufficiently difficult without such measures. Given this context, we have decided to leave the image data unadulterated. 


\section{Keywords}

qanon; savethechildren; conspiracy; image analysis. 